\title{
Association of novel single-nucleotide polymorphisms of the vascular endothelial growth factor-A gene with high-altitude adaptation in yak (Bos grunniens)
}

\author{
X.Y. Wu ${ }^{1,2}$, C.N. Liang ${ }^{1,2}$, X.Z. Ding ${ }^{1,2}$, X. Guo ${ }^{1,2}$, P.J. Bao ${ }^{1,2}$, M. Chu ${ }^{1,2}$, \\ W.B. Liu ${ }^{1,2}$ and P. Yan ${ }^{1,2}$ \\ ${ }^{1}$ Lanzhou Institute of Husbandry and Pharmaceutical Sciences, \\ Chinese Academy of Agricultural Science, Lanzhou, China \\ ${ }^{2}$ Key Laboratory of Yak Breeding Engineering Gansu Province, Lanzhou, China \\ Corresponding author: P. Yan \\ E-mail: pingyanlz@163.com
}

Genet. Mol. Res. 12 (4): 5506-5515 (2013)

Received August 6, 2012

Accepted March 12, 2013

Published November 18, 2013

DOI http://dx.doi.org/10.4238/2013.November.18.1

\begin{abstract}
Vascular endothelial growth factor-A gene $(V E G F-A)$ is a key regulator of angiogenesis and an endothelial cell mitogen that plays an important role in high-altitude adaptation. In this study, we detected 2 novel single-nucleotide polymorphisms (SNPs) of $V E G F-A$ by screening for genetic variation in 700 individuals of 3 domestic Chinese yak breeds - namely Gannan (GN), Datong (DT), and Tianzhu white (TZW) - using polymerase chain reaction-restriction fragment length polymorphism and DNA sequencing techniques. GN and DT yaks live at high altitude and TZW yaks live at low altitude on the Qinghai-Tibetan Plateau. SNP g.8430T $>C$ is located in intron 4 of $V E G F-A$. SNP g. $14853 \mathrm{G}>\mathrm{A}$ is located in the $3^{\prime}$ untranslated region of $V E G F-A$. Frequencies of the GA and AA genotypes and the A allele of SNP g.14853G $>$ A observed in GN and DT yaks were significantly higher than that in TZW yaks $(\mathrm{P}<0.01)$. No significant difference among the breeds was observed for SNP g.8430T $>$ C. The frequency
\end{abstract}


of haplotype TA was significantly higher $(\mathrm{P}<0.01)$, whereas the frequency of TG $(\mathrm{P}<0.01)$ was significantly lower in GN and DT yaks compared with that in TZW yaks. The 2 SNPs were in moderate linkage disequilibrium in GN and DT yaks, but not in TZW yaks. The fixation index $\left(F_{\mathrm{ST}}\right)$ pairwise value was significantly different among the breeds studied. The neutral test result indicated that the region between the 2 SNPs may have been subjected to positive or balancing selection, and the high-altitude hypoxia environment might be the main determinant for selection. These results suggest that $V E G F-A$ might contribute to the high-altitude adaptability of yak.

Key words: Vascular endothelial growth factor A (VEGF-A); Polymorphism; High-altitude adaptation; Hypoxia adaptation; Yak

\section{INTRODUCTION}

Vascular endothelial growth factor A (VEGF-A), also called VEGF, belongs to the VEGF family that also includes VEGF-B, VEGF-C, VEGF-D, VEGF-E, and placenta growth factor (Clauss, 2000). It was first identified in media conditioned with bovine pituitary follicular cells (Ferrara et al., 2003). VEGF-A is implicated in processes such as angiogenesis, vasculogenesis, and vascular endothelial cell division and in diseases such as cancer and diabetes (Greb et al., 1999; Shibuya and Claesson-Welsh, 2006; Stringer, 2006; Thangarajaha et al., 2009).

Recent reports have indicated that VEGF-A might play an important role in hypoxia response. $V E G F-A$ is a hypoxia-induced gene that stimulates the development of new blood and increases blood vessel permeability (Beall, 2007; Nagy et al., 2008). Hypoxia triggers the expression of $V E G F-A$ as well as its receptors in human endothelial and tumor cells (Ferrara, 2003). In humans, VEGF-A increases vascular permeability in brain tumors (Machein et al., 1999 ) and participates in the pathogenesis of high-altitude cerebral edema (Xu and Severinghaus, 1998). In rats, $V E G F-A$ expression is upregulated during the first week of brain hypoxia (Christou et al., 1998). Hypoxia-induced upregulation of expression for $V E G F-A$ and its receptors has been observed in rat lung (Marti et al., 1998). These findings suggest that $V E G F-A$ might be a candidate gene for high-altitude adaptation.

Yak (Bos grunniens) is a unique domestic animal that lives on the Qinghai-Tibetan Plateau at altitudes between 2500 and $6000 \mathrm{~m}$. It is a primary source of meat, milk, and hair in this area of Tibet. Owing to its adaptation to high-altitude environments and its high level of endurance for work, yak is a model animal for understanding the molecular basis of hypoxia adaptation. Accumulating research has focused on its singular adaptability to high altitude. Compared with cow and bull, yak has higher red blood cell (RBC) count, hemoglobin ( $\mathrm{Hb}$ ) content, and hematocrit (HCT) and more smooth muscle in pulmonary and bronchial arteries (Li et al., 2006; Ma et al., 2011). Yak also has pulmonary artery endothelial cells that are much longer, wider, and rounder than those in domestic cow (Miquerol et al., 2000; Wang et al., 2006). In response to hypoxia, $V E G F-A$ content increases in the intrapulmonary conducting airways and pulmonary arteries of 1-day-old yaks (He and Cui, 2008). Hence, VEGF-A might play a key role in high-altitude adaptation, but its genetic background remains unknown. 
In this study, we analyzed the polymorphisms of $V E G F-A$ in 3 yak breeds from different elevations to evaluate the relationship between $V E G F-A$ polymorphism and high-altitude adaptation.

\section{MATERIAL AND METHODS}

\section{Animals}

A total of 700 blood samples were obtained from 3 domestic yak breeds living at various altitudes. These breeds included Gannan (GN; $N=187)$, Datong (DT; $N=228)$ and Tianzhu white (TZW; $\mathrm{N}=288$ ). GN and DT yaks live at high altitude, and TZW yaks live at low attitude on the Qinghai-Tibetan Plateau (Figure 1). Genomic DNA was extracted with a genomic DNA isolation kit (TIANGENE, Beijing, China) according to manufacturer instructions.

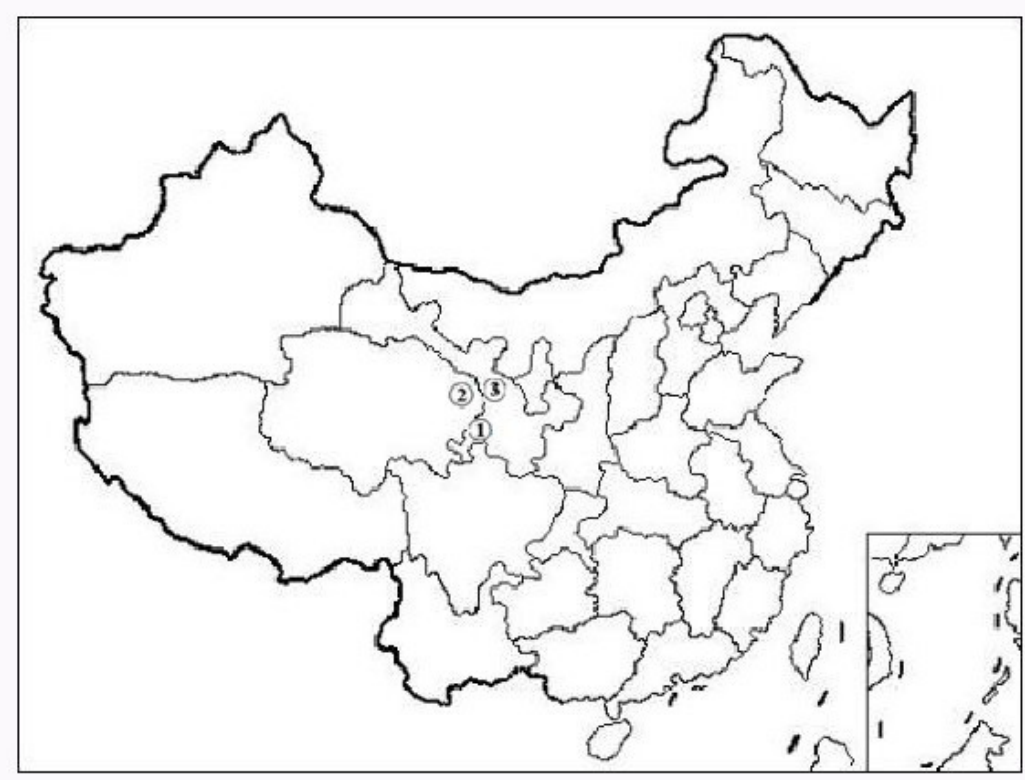

(1) Gannan County, Gansu Province, P. R. China Altitude $3600 \mathrm{~m}$

(2) Datong County, Qinghai Province, P. R. China Altitude $3100 \mathrm{~m}$

(3) Tianzhu County, Gansu Province, P. R. China Altitude $2700 \mathrm{~m}$

Figure 1. Geographic locations of the three breeds.

\section{DNA pool and polymerase chain reaction (PCR) amplification}

DNA samples from 50 randomly selected individuals were pooled to a final concentration of $100 \mathrm{ng} / \mu \mathrm{L}$. To examine the variation of $V E G F-A$, we used 6 pairs of PCR primers (Table 1) to amplify the yak $V E G F-A$ gene (Pang et al., 2011). 


\begin{tabular}{|c|c|c|c|}
\hline Locus & Position (bp) (NC_007324) & Primer sequence (5'-3') & Annealing temperature $\left({ }^{\circ} \mathrm{C}\right)$ \\
\hline $\mathrm{P} 1$ & $8-591$ & $\begin{array}{l}\text { F: 5' GACGGACAGACAGACAGACACC 3' } \\
\text { R: 5' AGGAGAAGGGGACAACAGAGG 3' }\end{array}$ & 61.6 \\
\hline $\mathrm{P} 2$ & $3521-3704$ & $\begin{array}{l}\text { F: 5' CTGCCGCTGCCCATTCTT 3' } \\
\text { R: 5' CCAACAGACCTTCCCACTCATC 3' }\end{array}$ & 58.1 \\
\hline P3 & 6661-6980 & $\begin{array}{l}\text { F: 5' CCTTTCCCGTGGTGGTTAC 3' } \\
\text { R: 5' CACCTGCATGGTGATGTTGA 3' }\end{array}$ & 62 \\
\hline P4 & $8217-8461$ & $\begin{array}{l}\text { F: 5' TCACCATCTGAACGCCTCT 3' } \\
\text { R: 5' CTCCATCCCACTGCTGCTA 3' }\end{array}$ & 59.8 \\
\hline P5 & $12380-12706$ & $\begin{array}{l}\text { F: 5' TCTTGTCTTCCGCTGTGGCAT 3' } \\
\text { R: 5' CTCTGACTTGCTCGCCCTCTG 3' }\end{array}$ & 64.3 \\
\hline P6 & $14709-14949$ & $\begin{array}{l}\text { F: 5' TGGAGGCTAGGACTGTGCTTT 3' } \\
\text { R: 5' GCGGCTATGGGTAGTTCTGTG 3' }\end{array}$ & 61.6 \\
\hline
\end{tabular}

Exons 1, 2, 3, 4, 5, and 6 are located at base pairs, 1-410, 3557-3608, 6781-6977, 8265-8301, 12545-12676, 1484115282 respectively (GenBank accession No. NC_007324).

The 15- $\mu \mathrm{L}$ PCR solution contained $50 \mathrm{ng}$ DNA template, $0.50 \mathrm{mM}$ deoxyribonucleotide triphosphates, $3 \mathrm{mM} \mathrm{MgCl}$, and $0.75 \mathrm{U}$ Taq DNA polymerase (TIANGENE, Beijing, China). PCR was performed using the following program: $94^{\circ} \mathrm{C}$ for 5 min followed by 35 cycles of $94^{\circ} \mathrm{C}$ for $40 \mathrm{~s}$, annealing at $61.6,58.1,62,59.8,64.3$, and $61.6^{\circ} \mathrm{C}$ (corresponding to 6 primer pairs) for $30 \mathrm{~s}$, and $72^{\circ} \mathrm{C}$ for $1 \mathrm{~min}$ and a final extension at $72^{\circ} \mathrm{C}$ for $10 \mathrm{~min}$. The PCR products were sequenced in both directions in an ABI 3730 DNA analyzer (Applied Biosystems, New York, USA). Sequences were analyzed using the DNASTAR 5.0 package (DNASTAR, Inc., Wisconsin, USA).

\section{Genotyping}

PCR-based restriction fragment length polymorphism (PCR-RFLP) analysis was performed. The RFLP conditions were as follows: $5 \mu \mathrm{L}$ PCR products was digested with $3 \mathrm{U}$ restriction enzymes (MBI Fermentas, Massachusetts, USA) at specific incubation temperatures overnight following supplier instructions. The resulting fragments were detected on $2.0-3.0 \%$ agarose gels.

\section{Statistical analysis}

Statistical analyses were carried out with SPSS 13.0 software for Windows (SPSS, Chicago, USA). Allele and genotype frequencies among groups were compared using the chisquare test. The haplotypes and linkage disequilibrium for each pair of segregating sites and the exact $\mathrm{P}$ value of Hardy-Weinberg equilibrium (HWE) for multiple alleles were assessed using SHEsis (Shi and He, 2005). The pairwise fixation index $\left(F_{\mathrm{ST}}\right)$ between population and neutrality tests (Tajima's $D$ test [Tajima, 1989], Fu and Li's $D$ and $F$ [Fu and Li, 1993], and Fu's $F_{\mathrm{S}}$ test [Fu, 1997]) were calculated using Arlequin 3.1 (Excoffier et al., 2005) and DnaSP (version 5.0) (Librado and Rozas, 2009).

\section{RESULTS}

\section{SNPs of $V E G F-A$ in 3 yak breeds}

Comparison between the sequence of bovine VEGF-A (GenBank accession No. 
NC_007324) and the pooled yak DNA samples revealed 2 SNPs, g.8430T $>$ C and g. 14853G $>$ A at $\bar{C} 4$ and $\mathrm{C} 6$ loci, respectively. SNP g.8430T $>\mathrm{C}$ was found in intron II of $V E G F-A$ and the SNP g.14853G $>$ A in exon V (3' untranslated region). The National Center for Biotechnology Information bovine SNP database did not contain these SNPs (g.8430T $>$ C, g.14853G $>$ A), so we concluded that they are specific to yak.

\section{Frequency distribution of genotype, haplotype, and linkage analysis}

SNP g.8430T $>C$ was genotyped using PCR-RFLP with HinII (MBI Fermentas). The result identified three genotypes (TT, TC, CC; Figure 2A, B). Genotypes of SNP g.8430T $>$ C were all in HWE. No significant differences in the allelic and genotypic distributions of SNP g.8430 $>\mathrm{C}$ were observed among the 3 breeds (Table 2).

A
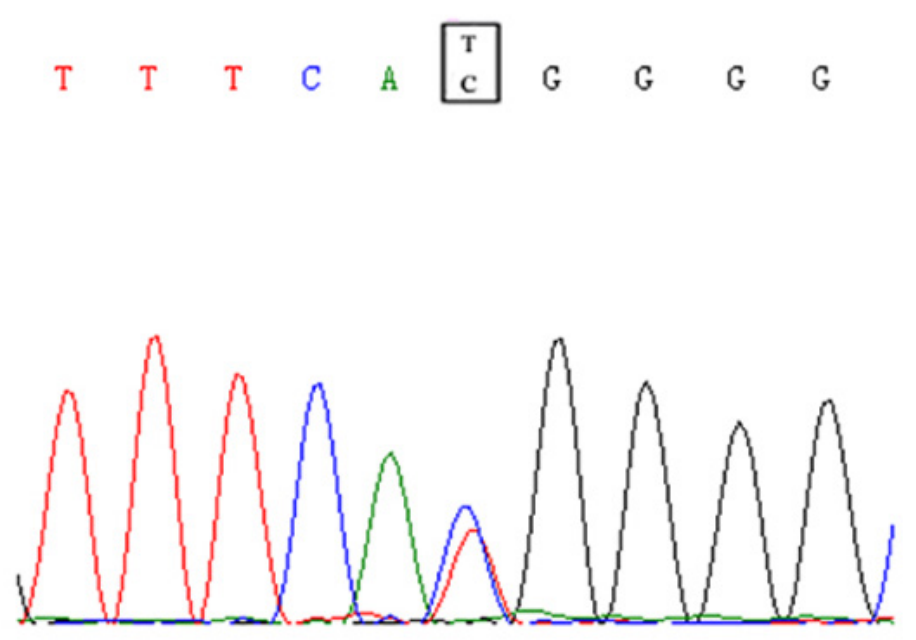

B bp

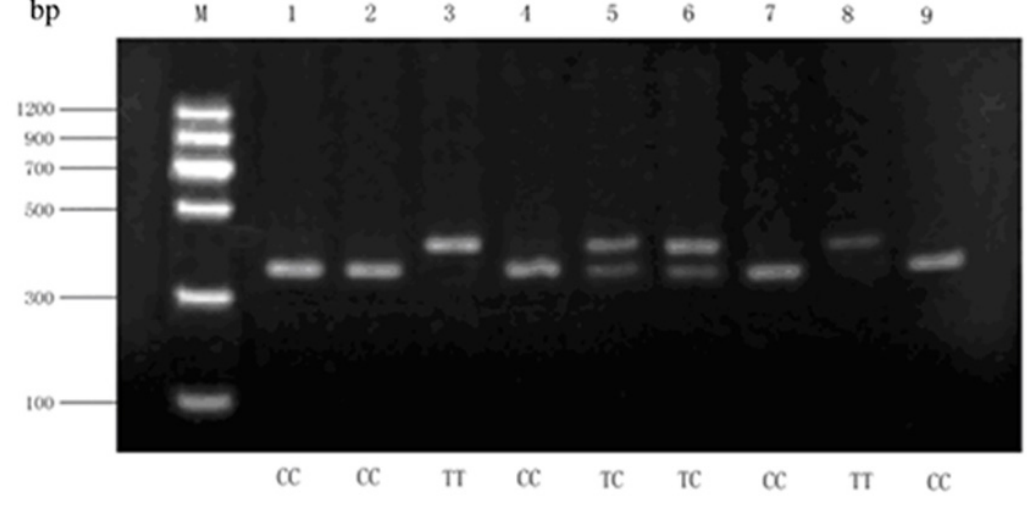

Figure 2. A. Sequencing maps at position of SNP g.8430T $>$ C in the yak $V E G F-A$ gene. B. Electrophoresis patterns of HinIII PCR-RFLP analysis of the yak $V E G F-A$ gene. 
Table 2. Allele and genotype distribution of SNP g.8430T $>\mathrm{C}$ in the three yak breeds.

\begin{tabular}{|c|c|c|c|c|c|}
\hline \multirow[t]{2}{*}{ Breeds } & \multicolumn{3}{|c|}{ Genotypes (\%) } & \multicolumn{2}{|c|}{ Alleles (\%) } \\
\hline & $\mathrm{CC}$ & $\mathrm{CT}$ & $\mathrm{TT}$ & $\mathrm{C}$ & $\mathrm{T}$ \\
\hline $\mathrm{GN}(\mathrm{N}=184)$ & $108(58.7 \%)$ & $50(27.1 \%)$ & $26(14.1 \%)$ & $266(72.3 \%)$ & $102(27.7 \%)$ \\
\hline DT $(\mathrm{N}=228)$ & $113(49.6 \%)$ & $87(38.2 \%)$ & $28(12.3 \%)$ & $313(68.8 \%)$ & $143(31.4 \%)$ \\
\hline TZW $(\mathrm{N}=288)$ & $153(53.1 \%)$ & $108(37.5 \%)$ & $27(9.4 \%)$ & $414(71.9 \%)$ & $162(28.1 \%)$ \\
\hline
\end{tabular}

Values with small (a and b) and capital (A and B) superscript letters within the same row in the same locus denote significant difference at $\mathrm{P}<0.05$ and $\mathrm{P}<0.01$.

To genotype SNP g.14853G $>$ A, we obtained PCR products of 241 bp with primer pair C6 (see Table 1) and digested them with StyI (MBI Fermentas). The result identified three genotypes (GG, GA, AA; Figure 3A, B). Genotypes at SNP g. 14853G>A were all in HWE. Both allelic and genotypic distributions were significantly different among the 3 yak breeds (Table 3). Frequencies of the GA and AA genotypes and the A allele observed in GN and DT yaks were significantly higher than that in TZW yaks $(\mathrm{P}<0.01)$.

A
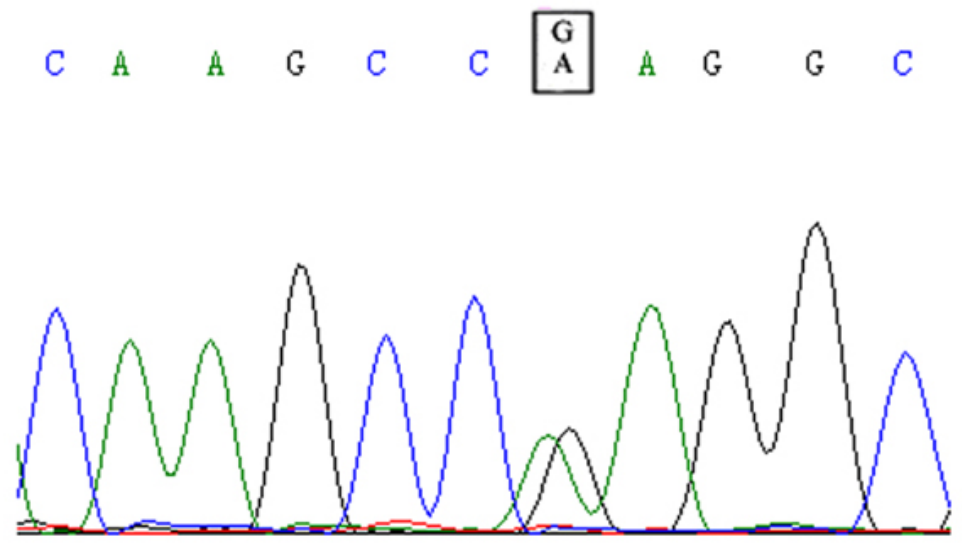

B

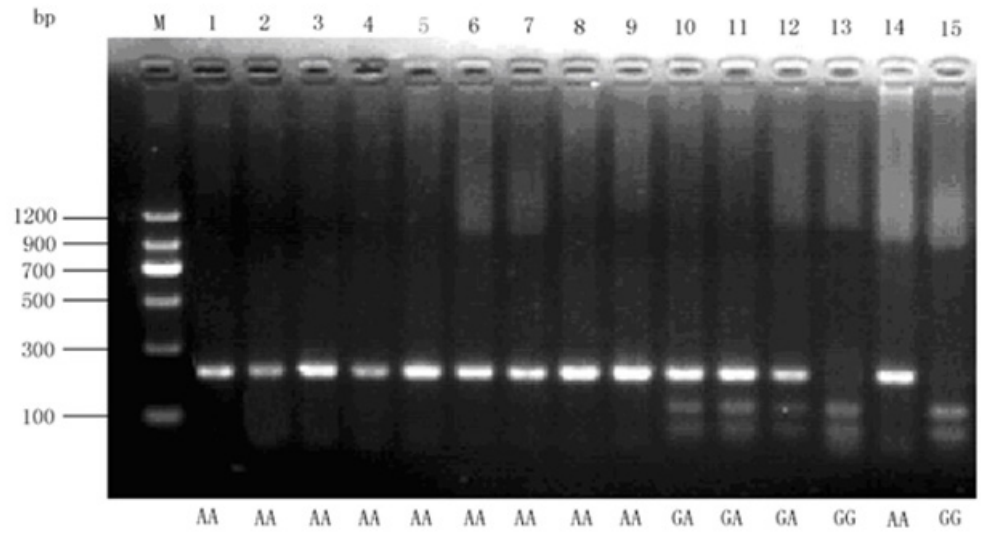

Figure 3. A. Sequencing maps at position of SNP g.14853G $>$ A in the yak $V E G F-A$ gene. B. Electrophoresis patterns of StyI PCR-RFLP analysis of the yak VEGF-A gene. 
Table 3. Allele and genotype distribution of SNP g.14853G $>$ A in the three yak breeds.

\begin{tabular}{|c|c|c|c|c|c|}
\hline \multirow[t]{2}{*}{ Breeds } & \multicolumn{3}{|c|}{ Genotypes (\%) } & \multicolumn{2}{|c|}{ Alleles (\%) } \\
\hline & GG & GA & AA & G & A \\
\hline $\mathrm{GN}(\mathrm{N}=184)$ & $98(53.3 \%)$ & $74(40.2 \%)^{\mathrm{A}}$ & $12(6.5 \%)^{\mathrm{A}}$ & $318(73.4 \%)$ & $138(26.6 \%)^{\mathrm{A}}$ \\
\hline DT $(\mathrm{N}=228)$ & $110(48.2 \%)$ & $98(43 \%)^{\mathrm{A}}$ & $20(8.8 \%)^{\mathrm{A}}$ & $270(69.7 \%)$ & $98(30.3 \%)^{\mathrm{A}}$ \\
\hline TZW $(\mathrm{N}=288)$ & $208(72.2 \%)$ & $76(26.4 \%)^{\mathrm{B}}$ & $4(1.4 \%)^{\mathrm{B}}$ & $492(85.4 \%)$ & $84(14.6 \%)^{\mathrm{B}}$ \\
\hline
\end{tabular}

Values with small (a and b) and capital (A and B) superscript letters within the same row in the same locus denote significant difference at $\mathrm{P}<0.05$ and $\mathrm{P}<0.01$, respectively.

Haplotype-based analysis showed significant differences in the haplotype distribution among the 3 yak breeds $(\mathrm{P}<0.01)$. Compared with that in TZW yaks, the frequencies of haplotype TA $(\mathrm{P}<0.01)$ was significantly higher and the frequency of TG $(\mathrm{P}<0.01)$ was significantly lower in GN and DT yaks (Table 4). The 2 VEGF-A variants were in moderate linkage disequilibrium in GN and DT yaks but not in TZW yaks (Table 5).

Table 4. Haplotype distribution of SNPs (g.8430T $>$ C, g. $14853 \mathrm{G}>$ A ) in the three yak breeds.

\begin{tabular}{llll}
\hline Haplotype & GN $(\%)$ & DT (\%) & TZW (\%) \\
SNPs: g.8211C>A, g.13374G $>$ A & & 57 & 61.3 \\
\hline CG & 60.3 & 11.6 & 10.6 \\
CA & 12 & $12.7^{\mathrm{B}}$ & $24.1^{\mathrm{A}}$ \\
TG & $13.3^{\mathrm{B}}$ & $18.6^{\mathrm{A}}$ & $4^{\mathrm{B}}$ \\
TA & $14.4^{\mathrm{A}}$ & \\
\hline
\end{tabular}

Values with small ( $\mathrm{a}$ and $\mathrm{b}$ ) and capital $(\mathrm{A}$ and $\mathrm{B})$ superscript letters within the same row in the same locus denote significant difference at $\mathrm{P}<0.05$ and $\mathrm{P}<0.01$, respectively.

Table 5. Analysis of linkage disequilibrium of the two polymorphisms in the three yak breeds.

\begin{tabular}{lcr}
\hline Breeds & D' $^{\prime}$ & $\mathrm{r}^{2}$ \\
\hline GN & 0.431 & 0.153 \\
DT & 0.491 & 0.176 \\
TZW & 0.276 & 0.047 \\
\hline
\end{tabular}

\section{Neutral test}

Pairwise fixation index (FST) was significantly different among the 3 yak breeds. No significant difference was found between GN and DT yaks. To investigate further the regions between the 2 SNPs, 4 statistics (Tajima's D test, Fu and Li's F and D, and Fu's F test) were calculated to detect selective signals. The results revealed that the region may have been subjected to positive or balancing selection (Table 6).

Table 6. Neutral test per breed.
\begin{tabular}{lcccc}
\hline Breeds & Tajima's D & Fu-Li's D & Fu-Li's F & Fu's Fs \\
\hline GN & 1.95845 & 0.80615 & 1.18327 & 1.63703 \\
DT & 2.26733 & 0.80615 & 1.02510 & 2.05115 \\
TZW & 1.50187 & 0.80615 & 0.95514 & 1.27973 \\
\hline
\end{tabular}




\section{DISCUSSION}

During the past 10,000 years, yak has occupied almost all available environments in the Qinghai-Tibetan Plateau, and physiological adaptations have contributed to their success in surviving rigorous environmental conditions. Throughout their evolutionary history, yak populations have been exposed to myriad pressures from natural selection. Confronted stresses (selective pressures) are important factors influencing the fate of variations in the genetic background of the species. The genetic changes arising from variable metabolic efficiency, resistance to pathogens, and even the preferences of potential mates in combination with natural selection produce adapted phenotypic patterns. High-altitude hypoxia is one environmental stress that determines the survival of yak. Research has established that when altitude increases and the partial pressure of oxygen decreases, the oxygen content in the blood of yaks decreases. Tissue hypoxia is thought to upregulate a series of local factors that promote RBC count, $\mathrm{Hb}$ content, and HCT increase to meet bodily oxygen demand. Jiang et al. (1991) have suggested that the number of alveoli, RBC count, $\mathrm{Hb}$ content, and HCT are higher in high-altitude yaks than they are in yaks living at lower altitude. We speculate that high-altitude yaks have better adaptability in a hypoxic environment.

In the present study, we analyzed the alleles, genotypes, and haplotypes of $2 \mathrm{VEGF}$ $A$ polymorphisms in 3 yak breeds. Significant differences in allele and genotype frequencies of SNP g.13374G $>$ A among the breeds were detected. The 2 SNPs were in moderate linkage disequilibrium in high-altitude yaks but not in low-altitude yaks, which indicated that $V E G F-A$ may be a rarely occurring recombination in high-altitude yaks and selection is likely a reason for the moderate linkage disequilibrium. The results of neutral selection were positive but not significant, and we suspect that the differentiation between high-altitude and low-altitude yaks is relatively young because some mutations with relevance to high-altitude adaptation are not spread completely throughout the yak population. The significant differences among these 3 yak breeds may be caused by the differences in the breeds, but the significantly higher frequency of the SNP g.13374G $>$ A A allele in GN and DT yaks, which have lived at high altitude for many generations, is likely the result of a long period of natural selection for environmental adaptation.

Tissue hypoxia can reportedly upregulate a series of local factors that contribute to angiogenesis and the growth of new capillary vessels, which increase delivery of both oxygen and energy substrates such as glucose. $V E G F-A$ expression is induced by hypoxia, and it is the critical angiogenic factor implicated in vasculogenesis. $V E G F-A$ is a potent mitogen for vascular endothelial cells and smooth muscle cells, and it can activate endothelial nitric oxide synthase to accumulate the synthesis of nitric oxide (Miquerol et al., 2000). Nitric oxide relaxes blood vessel and increases blood flow velocity, preventing pulmonary hypertension (Kroll and Waltenberger, 1998). Hypoxia also induces factors that regulate $V E G F-A$ messenger RNA stability by binding to sequences located in the 3' untranslated region (Claffey et al., 1998). Stability of $V E G F-A$ messenger RNA can greatly increase the half-life of what are normally short-lived transcripts (Misquitta et al., 2001). These findings illustrated the G to A substitution of in $V E G F-A$ provides better protection against hypoxia. These yaks inhabit an area of the plateau with a hypoxic environment. The better hypoxia adaptation of A allele carriers and hypoxic environment selection over a long period might result in the higher frequency of the A allele in the high-altitude yak population. 
Study of haplotypes has proved useful for a more comprehensive analysis of association results. Our results suggested that the haplotypes TA constructed by SNP g.14853G $>$ A may impart better adaptation capability in the hypoxic environment of the plateau.

We also found that the A allele and haplotype TA were observed in higher frequencies in DT yaks than in GN yaks, although the difference was not significant. This unexpected result occurs because the DT breed is an artificial cultivation with half the genes of wild yak (Bos mutus). Wild yak lives in an area $4000 \mathrm{~m}$ above the sea level in the Qinghai-Tibetan Plateau and displays the best adaptation for high-altitude hypoxia. The adaptation of this species is reflected in the elevated resting ventilation and high $\mathrm{Hb}$ concentration compared with that of bovines. This result might indicate that semi-wild yaks also have better adaptability than that of domestic yaks in a hypoxic environment.

In conclusion, our results indicate that $V E G F-A$ may have been subjected to positive or balancing selection in yak, and the SNP g.14853G $>$ A A allele and TA haplotypes might be advantageous for yak adaptation to high-altitude environments. Further research in different populations and larger sample sizes is required to elucidate further the relationship between the 2 polymorphisms and high-altitude adaptation.

\section{ACKNOWLEDGMENTS}

Research supported by the National Beef Cattle Industry Technology \& System (\#CARS-38), Special Fund for Agro-scientific Research in the Public Interest (Contract \#201003061), 'Five-twelfth' National Science and Technology Support Program (\#2012BAD13B05), the Great Project of Science and Technology of Gansu Province in China (Contract \#1102NKDA027), and the Natural Science Foundation of China (Grant \#31101702).

\section{REFERENCES}

Beall CM (2007). Detecting natural selection in high-altitude human populations. Respir. Physiol. Neurobiol. 158: 161-171.

Christou H, Yoshida A, Arthur V, Morita T, et al. (1998). Increased vascular endothelial growth factor production in the lungs of rats with hypoxia-induced pulmonary hypertension. Am. J. Respir. Cell Mol. Biol. 18: 768-776.

Claffey KP, Shih SC, Mullen A, Dziennis S, et al. (1998). Identification of a human VPF/VEGF 3' untranslated region mediating hypoxia-induced mRNA stability. Mol. Biol. Cell 9: 469-481.

Clauss M (2000). Molecular biology of the VEGF and the VEGF receptor family. Semin. Thromb. Hemost. 26: 561-569.

Excoffier L, Laval G and Schneider S (2005). Arlequin (version 3.0) an integrated software package for population genetics data analysis. Evol. Bioinform. Online 1: 47-50.

Ferrara N, Gerber HP and LeCouter J (2003). The biology of VEGF and its receptors. Nat. Med. 9: 669-676.

Fu YX and Li WH (1993). Statistical tests of neutrality of mutations. Genetics 133: 693-709.

Fu YX (1997). Statistical tests of neutrality of mutations against population growth, hitchhiking and background selection. Genetics 147: 915-925.

Gerber HP and LeCouter J (2003). The biology of VEGF and its receptors. Nat. Med. 9: 669-676.

Greb RR, Maier I, Wallwiener D and Kiesel L (1998). Vascular endothelial growth factor A (VEGF-A) mRNA expression levels decrease after menopause in normal breast tissue but not in breast cancer lesions. Br. J. Cancer. 81: 225-231.

He J-F and Cui Y (2008). Distribution of vascular endothelial growth factor in plateau yaks lung. Chin. Vet. Sci. 38: 10861088.

Jiang JC, Gama RZ and He ML (1991). Comparison of the tibetan plateau yak blood physiology value from different altitude. Chin. J. Anim. Vet. 22: 20-26.

Kroll J and Waltenberger J (1998). VEGF-A induces expression of eNOS and iNOS in endothelial cells via VEGF receptor-2 (KDR). Biochem. Biophys. Res. Commun. 252: 743-746.

$\mathrm{Li} \mathrm{L}$, Shen M and $\mathrm{Yu} \mathrm{H}$ (2006). Significance and determination of RBC, $\mathrm{Hb}$ and $\mathrm{Mb}$ in yak of various ages. Acta Ecol. 
Animalis Domastici 27: 51-54.

Librado P and Rozas J (2009). DnaSP v5: a software for comprehensive analysis of DNA polymorphism data. Bioinformatics 25: 1451-1452.

Ma XB, Cui Y, He J-F and Yang B (2011). Observations of the histological structure of adult yak bronchial arteries. Chin. Vet. Sci. 39: 261-265.

Machein MR, Kullmer J, Fiebich BL, Plate KH, et al. (1999). Vascular endothelial growth factor expression, vascular volume, and, capillary permeability in human brain tumors. Neurosurgery 44: 732-740.

Marti HH and Risau W (1998). Systemic hypoxia changes the organ-specific distribution of vascular endothelial growth factor and its receptors. PNAS 95: 15809-15814.

Miquerol L, Langille BL and Nagy A (2000). Embryonic development is disrupted by modest increases in vascular endothelial growth factor gene expression. Development 127: 3941-3946.

Misquitta CM, Iyer VR, Werstiuk ES and Grover AK (2001). The role of 3'-untranslated region (3'-UTR) mediated mRNA stability in cardiovascular pathophysiology. Mol. Cell Biochem. 224: 53-67.

Nagy JA, Benjamin L, Zeng H, Dvorak AM, et al. (2008). Vascular permeability, vascular hyperpermeability and angiogenesis. Angiogenesis 11: 109-119.

Pang Y, Lei C, Zhang C, Lan X, et al. (2011). Analysis of the genetic variation of vascular endothelial growth factor gene in three Chinese indigenous cattle breeds. Mol. Biol. Rep. 38: 3219-3225.

Shi YY and He L (2005). SHEsis, a powerful software platform for analyses of linkage disequilibrium, haplotype construction, and genetic association at polymorphism loci. Cell Res. 15: 97-98.

Shibuya M and Claesson-Welsh L (2006). Signal transduction by VEGF receptors in regulation of angiogenesis and lymphangiogenesis. Exp. Cell Res. 312: 549-560.

Stringer SE (2006). The role of heparan sulphate proteoglycans in angiogenesis. Biochem. Soc. Trans. 34: 451-453.

Tajima F (1989). Statistical method for testing the neutral mutation hypothesis by DNA polymorphism. Genetics 123: 585-595.

Thangarajaha H, Yaob D, Chang EI, Shi Y, et al. (2009). The molecular basis for impaired hypoxia-induced VEGF expression in diabetic tissues. PNAS 103: 13505-13510.

Wang DP, Li HG, Li YJ, Guo SC, et al. (2006). Hypoxia-inducible factor 1alpha cDNA cloning and its mRNA and protein tissue specific expression in domestic yak (Bos grunniens) from Qinghai-Tibetan plateau. Biochem. Biophys. Res. Commun. 348: 310-319.

Xu F and Severinghaus JW (1998). Rat brain VEGF expression in alveolar hypoxia: possible role in high-altitude cerebral edema. J. Appl. Physiol. 85: 53-57. 